\title{
Synthesis of 1-aroylmethylpyrroles as useful intermediates for further chemical transformation
}

\author{
Nikolaos Karousis, Konstantina Koriatopoulou, and George Varvounis* \\ Department of Chemistry, University of Ioannina, 45110 Ioannina, Greece \\ E-mail: gvarvoun@cc.uoi.gr
}

\begin{abstract}
The synthesis of 1-aroylmethylpyrroles from 2-bromo-1-(2-aminophenyl)ethan-1-one and monodi- or tri-substituted $1 \mathrm{H}$-pyrroles, has been investigated. The reactions take place at r.t. or $80{ }^{\circ} \mathrm{C}$ in DMF containing potassium carbonate. Reduction of 1-[2-(2-aminophenyl)-2-oxoethyl]-4bromo-1H-pyrrole-2-carbaldehyde with sodium borohydride gave racemic 1-(2-aminophenyl)-2[4-bromo-2-(hydroxymethyl)-1H-pyrrol-1-yl]ethanol.
\end{abstract}

Keywords: Pyrroles, 1-aroylmethylpyrroles, alkylation, reduction

\section{Introduction}

The importance of 1-aroylmethylpyrroles stems from the fact that they constitute the central structural component in marine natural products such as the Lamellarins ${ }^{1}$ and are precursors to the closely related Lukianols. ${ }^{2}$ Moreover, 1-aroylmethylpyrroles are useful precursors in the synthesis of pyrrolo[2,1- $b][3]$ benzazepines $^{1}$ and pyrrolo[2,1-b][1,3] benzothiazepines ${ }^{3}$, compounds that possess interesting biological properties. Several syntheses of 1 aroylmethylpyrroles have been reported. Belanger and co-workers ${ }^{3}$ synthesised methyl 1-(2-oxo2-arylethyl)-1 $\mathrm{H}$-pyrrole-2-carboxylates by reacting 2-bromo-1-arylethanones and methyl $1 \mathrm{H}$ pyrrole-2-carboxylates at ambient temperature in DMF containing potassium carbonate. Artico and co-workers ${ }^{5}$ synthesised 1-aryl-2-(1H-pyrrol-1-yl)ethanones from 2-amino-1-arylethanone hydrochlorides and 2,5-dimethoxytetrahydrofuran. These reactions were carried out by heating briefly in DMF. En route to pyrrolobenzothiazepines, Campiani et al. ${ }^{4,6}$ synthesised several 1aryl-2-(1H-pyrrol-1-yl)ethanones by the same method, except that the reactions were carried out in boiling acetic acid containing aqueous sodium acetate. 


\section{Results and Discussion}

Herein we describe the preparation of 1-aroylmethylpyrroles 3a-h by reacting 2-bromo-1-(2aminophenyl)ethan-1-one $\mathbf{1}$ with the appropriately substituted pyrroles 2a-h in DMF containing potassium carbonate (Scheme 1). The substrates used, reaction time, temperature and yield of products are given in Table 1.<smiles>Nc1ccccc1C(=O)CBr</smiles>

1<smiles>[R]c1[nH]c([R])c([R])c1[R]</smiles>

2<smiles>[R]c1c([R])c([R])n(CC(=O)c2ccccc2N)c1[R]</smiles>

3

Scheme 1. Reagents and conditions: (i) $\mathrm{K}_{2} \mathrm{CO}_{3}$, DMF, r.t. or $80{ }^{\circ} \mathrm{C}$.

Table 1. Conversion of 2-bromo-1-(2-aminophenyl)ethan-1-one 1 into 1-aroylmethylpyrroles 3a-h

\begin{tabular}{cccccccc}
\hline Pyrrole & Product & $\mathrm{R}^{1}$ & $\mathrm{R}^{2}$ & $\mathrm{R}^{3}$ & $\mathrm{R}^{4}$ & $\begin{array}{c}\mathrm{Temp} \\
\left({ }^{\circ} \mathrm{C}\right)\end{array}$ & $\begin{array}{c}\text { Time } \\
(\mathrm{h})\end{array}$ \\
\hline $\mathbf{2 a}$ & $\mathbf{3 a}$ & $\mathrm{CHO}$ & $\mathrm{H}$ & $\mathrm{H}$ & $\mathrm{H}$ & 22 & 3 \\
$\mathbf{2 b}$ & $\mathbf{3 b}$ & $\mathrm{CHO}$ & $\mathrm{H}$ & $\mathrm{CHO}$ & $\mathrm{H}$ & r.t. & 3 \\
$\mathbf{2 c}$ & $\mathbf{3 c}$ & $\mathrm{CHO}$ & $\mathrm{H}$ & $\mathrm{Br}$ & $\mathrm{H}$ & 80 & 16 \\
$\mathbf{2 d}$ & $\mathbf{3 d}$ & $\mathrm{CHO}$ & $\mathrm{CH}=\mathrm{CHCONM}{ }_{2}$ & $\mathrm{H}$ & $\mathrm{H}$ & 80 & 16 \\
$\mathbf{2}$ & $\mathbf{3 e}$ & $\mathrm{CO}_{2} \mathrm{Me}$ & $\mathrm{H}$ & $\mathrm{H}$ & $\mathrm{H}$ & r.t. & 48 \\
$\mathbf{2 f}$ & $\mathbf{3 f}$ & $\mathrm{CO}_{2} \mathrm{Me}$ & $\mathrm{H}$ & $\mathrm{Br}$ & $\mathrm{H}$ & r.t. & 48 \\
$\mathbf{2 g}$ & $\mathbf{3 g}$ & $\mathrm{CO}_{2} \mathrm{Me}$ & $\mathrm{H}$ & $\mathrm{Br}$ & $\mathrm{Br}$ & r.t. & 48 \\
$\mathbf{2 h}$ & $\mathbf{3 h}$ & $\mathrm{CO}_{2} \mathrm{Me}$ & $\mathrm{H}$ & $\mathrm{CH}=\mathrm{CHCONMe}_{2}$ & $\mathrm{H}$ & 80 & 16 \\
\hline
\end{tabular}

The precursor to 2-bromo-1-(2-aminophenyl)ethan-1-one $\mathbf{1}$ is 2-bromo-1-(2-nitrophenyl)ethan-1-one. The latter was prepared by dropwise addition of bromine solution to 2nitroacetophenone in chloroform following basically the procedure by Andreani et al. ${ }^{7}$ who did not isolate the material but reacted it straight with 2 -aminothiazole. In our hands the compound was isolated in $83 \%$ yield. 2-Bromo-1-(2-nitrophenyl)ethan-1-one was reduced to compound 1 by copper powder in sulfuric acid according to the method of Grehm. ${ }^{8}$ Any attempt to reduce the compound by other means including $\mathrm{Zn}$ or $\mathrm{Fe}$ and acid, $\mathrm{SnCl}_{2}$ or catalytic hydrogenation, failed. $1 H$-Pyrrole-2-carbaldehyde 2a was prepared from freshly distilled $1 H$-pyrrole by VilsmeierHaack formylation. ${ }^{9}$ Freshly distilled $1 H$-pyrrole was also used to prepare $1 H$-pyrrole-2,4dicarbaldehyde $\mathbf{2 b}$, by reacting with oxalyl chloride in DMF and then formylating the 
intermediate $N$-[(1H-pyrrol-2-yl)methylene]- $N$-methylmethanaminium chloride by reacting with dichloro(methoxy)methane with aluminium trichloride as catalyst. ${ }^{10}$ 4-Bromo-2-formyl- $1 H$ pyrrole $2 \mathrm{c}$ was synthesised by the method of Sonnet ${ }^{11}$ that is, bromination of the ternary iminium salt, obtained from $1 \mathrm{H}$-pyrrole-2-carbaldehyde $\mathbf{2 a}$ and pyrrolidinium perchlorate in benzene, followed by mild hydrolysis. The synthesis of enamides $\mathbf{2 d}$ and $\mathbf{7}$ was accomplished in two steps. In the first step Friedel-Crafts formylation of prop-2-enamide 4, prepared previously in our laboratory, ${ }^{12}$ with 1,1-dichloro-2-methoxyethane in the presence of aluminium trichloride gave a mixture that separated by column chromatography into (E)-3-(2-formyl)-2-enamide 5 and $(E)-3$ (5-formyl)-2-enamide 6 in 53 and 11\% yield, respectively. A characteristic difference in the ${ }^{1} \mathrm{H}$ NMR spectra of compounds 5 and $\mathbf{6}$ is the expected large coupling constant between $\mathrm{H}-4$ and $\mathrm{H}-$ $5\left(J_{4,5}=3.2 \mathrm{~Hz}\right)$ of 5 and a much smaller coupling constant between H-2 and H-4 (not measured by the $400 \mathrm{MHz}$ instrument) of $\mathbf{6}$. In the second step compounds 5 and $\mathbf{6}$ were detosylated by treatment with a methanolic solution of potassium carbonate, to yield corresponding enamides 2d and 7 in 88 and $82 \%$ yield, respectively (Scheme 2).

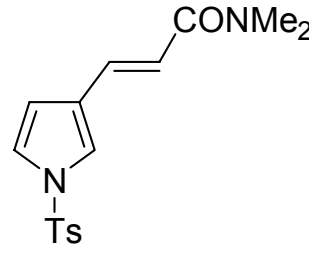

4

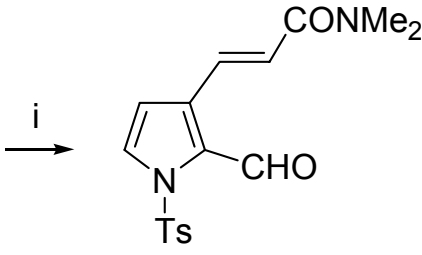

5
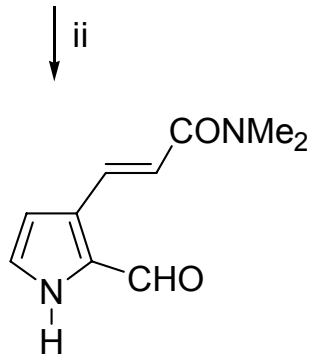

2d

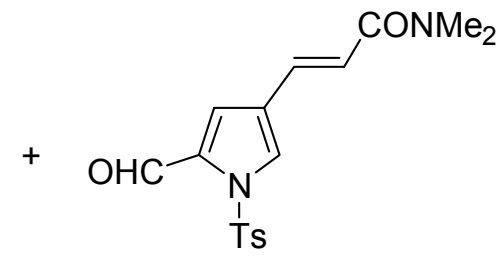

6

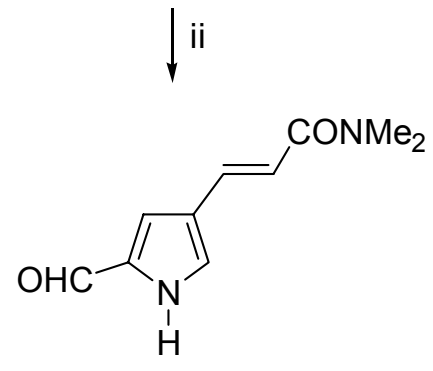

7

Scheme 2. Reagents and conditions: (i) $\mathrm{Cl}_{2} \mathrm{CHOMe}, \mathrm{ClCH}_{2} \mathrm{CH}_{2} \mathrm{Cl}, \mathrm{AlCl}_{3}, 0^{\circ} \mathrm{C}, 1 \mathrm{~h}$., (ii) $\mathrm{K}_{2} \mathrm{CO}_{3}$, $\mathrm{MeOH}, 1 \mathrm{~h}$.

The synthesis of $1 H$-pyrrole-2-carboxylic acid ethyl ester $2 \mathbf{e}$ took place according to Harbuck and Rapoport ${ }^{13 a}$ by trichloroacetylating $1 H$-pyrrole with trichloacetyl chloride to 2,2,2-trichloro1-(1H-pyrrol-2-yl)ethanone and then esterifying with a methanolic solution of sodium methoxide. Compound 2e was brominated with one equivalent of bromine in DMF containing potassium carbonate to 4-bromo- $1 H$-pyrrole-2-carboxylic acid ethyl ester $2 \mathrm{f}$ in $85 \%$ yield. ${ }^{14}$ On the other hand, methyl 4,5-dibromo- $1 H$-pyrrole-2-carboxylate $\mathbf{2 g}$ was obtained by brominating 2,2,2-trichloro-1-(1H-pyrrol-2-yl)ethanone with two equivalents of bromine and then esterifying 
2,2,2-trichloro-1-(4,5-dibromo-1H-pyrrol-2-yl)ethanone ${ }^{15}$ with methanolic sodium methoxide. Compound $\mathbf{2 g}$ was isolated in near quantitative yield from the last step. A literature report describes the preparation of $\mathbf{2} \mathbf{g}$ by the reaction of bromo ester $\mathbf{2} \mathbf{f}$ with NBS in DMF, albeit in only $29 \%$ yield. ${ }^{13 \mathrm{~b}}$ The Horner-Wadsworth-Emmons reaction was used to synthesise propenoate $\mathbf{2 h}$ by reacting phosphonate mono-carbanion, produced from dimethylcarbamoylmethylphosphonic acid diethyl ester and slight excess of $\mathrm{NaH}$ in THF, with 4-formyl- $1 H$ pyrrole-2-carboxylic acid methyl ester. The latter was prepared according to Rapoport and coworkers, ${ }^{16}$ in two steps, from 2,2,2-trichloro-1-(1H-pyrrol-2-yl)ethanone by Friedel-Crafts formylation with 1,1-dichloro-2-methoxyethane in the presence of aluminium trichloride and then esterification of 5-(2,2,2-trichloroacetyl)-1H-pyrrole-3-carbaldehyde with a methanolic solution of sodium methoxide.

The 1-aroylmethylpyrroles 3a-h obtained as described above (Table 1) are useful precursors for further chemical transformation. One of the goals set forth at the beginning of this work was to study the intramolecular cyclisation of these compounds leading to pyrrolobenzodiazocines, analogues of the naturally occurring pyrrolo[2,1-c][1,4]benzodiazepine family of antibiotics. A preliminary study on the cyclodehydration of $\mathbf{3 c}$ to pyrrolobenzodiazocine $\mathbf{8}$ has been undertaken. Compound 3c was subjected to several literature methods for cyclising aminoaldehydes, including an intramolecular aza-Wittig reaction (dibromotriphenylphosphine and triethylamine), refluxing in ethanol or 2-methoxyethanol, in the presence of molecular sieves and Dean-Stark conditions (toluene and a catalytic amount of $p$-toluenesulfonic acid) all resulting in unreacted starting material (Scheme 3). The resistance of compound $\mathbf{3 c}$ to cyclodehydration may be due to its non-favourable conformation, possibly because of the keto group. One further attempt to cyclise compound $\mathbf{3 c}$ was by reduction with sodium borohydride. It was hoped that selective reduction of $\mathbf{3 c}$ would give the less conformationally constrained alcohol $\mathbf{1 0}$ where interaction between the amino and aldehyde groups of $\mathbf{1 0}$ would lead to pyrrolobenzodiazocine 11. Instead, non-selective reduction of $\mathbf{3 c}$ led to racemic dialcohol 9. 
<smiles>CC(C)(C)C(C)(C)C</smiles>

8<smiles>CCC(C)(C)CC(C)(C)Cn1cc(Br)cc1C(O)Cn1cc(Br)cc1C(O)Cn1cc(Br)cc1N</smiles>

10<smiles>OC1Cn2cc(Br)cc2C=Nc2ccccc21</smiles>

11

Scheme 3. Reagents and conditions: (i) (a) $\mathrm{Ph}_{3} \mathrm{PBr}_{2}, \mathrm{Et}_{3} \mathrm{~N}, \mathrm{CH}_{2} \mathrm{Cl}_{2}$, (b) $\mathrm{EtOH}$ or $\mathrm{MeO}\left(\mathrm{CH}_{2}\right)_{2} \mathrm{OH}$, $3 \AA ̊$ molecular sieves, reflux or (c) toluene, TsOH, Dean-Stark, (ii) $\mathrm{NaBH}_{4}, \mathrm{Et}_{2} \mathrm{O}, 48 \mathrm{~h}$.

At the present time we are investigating further the chemistry of the 1-aroylmethylpyrroles in order to synthesise a large variety of derivatives and prepare a profile of their biological activity. We are also examining the possibility of using the compounds as precursors to the pyrrolobenzodiazocine, 2,3-dihydro- $1 H$-quinolin-4-one and 3,4-dihydropyrrolo[2,1-c][1,4]oxazin-1-one ring systems.

\section{Conclusions}

We have described an efficient method of synthesising 1-aroylmethylpyrroles from 2-bromo-1(2-aminophenyl)ethan-1-one and mono-, di- or tri-substituted $1 H$-pyrroles. These compounds are potentially useful as intermediates for further functional group transformation in heterocyclic synthesis and as scaffolds for the creation of libraries.

\section{Experimental Section}

General Procedures. Melting points were taken on a Büchi 510 apparatus and are uncorrected. Infrared spectra were recorded on a Perkin-Elmer 257 spectrometer, as Nujol mulls and liquids 
between sodium chloride discs. Nuclear magnetic resonance spectra were measured at $300 \mathrm{MHz}$ on Brüker AC 300 spectrometer, at $250 \mathrm{MHz}$ on a Brüker AM 250 spectrometer or at $400 \mathrm{MHz}$ on a Brüker AMX 400 spectrometer using tetramethylsilane as internal standard. Mass spectra were obtained using a JEOL JMS-AX 505W or a Bruker Apex III high resolution instruments Analytical TLC was carried out on Fluka silica gel 60 F254. Preparative flash chromatography was carried out for all separations using Merck 9385 silica gel. Solvents and reagents were used as received from the manufacturers, except for dichloromethane, methanol, tetrahydrofuran, ethyl acetate and hexane that were purified and dried according to standard procedures.

\section{General Procedure for the preparation of 1-aroylmethylpyrroles (3a-h)}

To a stirred mixture of the appropriate pyrrole $\mathbf{2 a - h}(3 \mathrm{mmol})$ (Table 1$)$ and potassium carbonate (0.83 g, $6 \mathrm{mmol})$ in dry DMF $(5 \mathrm{~mL})$ under an atmosphere of argon, was added 2-bromo-1-(2aminophenyl)ethan-1-one $1(0.64 \mathrm{~g}, 3 \mathrm{mmol})$. The reaction mixture was stirred at room temperature or at $80{ }^{\circ} \mathrm{C}$ for 3,16 or $48 \mathrm{~h}$, cooled, poured into ice-cold water $(25 \mathrm{~mL})$ and extracted with EtOAc $(3 \times 10 \mathrm{~mL})$. The combined organic extracts were washed with brine $(10$ $\mathrm{mL})$ and dried $\left(\mathrm{Na}_{2} \mathrm{SO}_{4}\right)$. The solvent was evaporated under vacuo to give, either an oily residue which was triturated with diethyl ether to give a solid or a solid residue, which was then purified either by column chromatography followed by recrystallisation or by recrystallisation, to afford the product.

1-[2-(2-Aminophenyl)-2-oxoethyl]-1H-pyrrole-2-carbaldehyde (3a). Colourless microcrystals (recrystallised from toluene); (0.42 g, 62\%); mp 159-160 ${ }^{\circ} \mathrm{C}$. IR (Nujol, $\left.\mathrm{cm}^{-1}\right): 3475,3375,1660$, 1640; ${ }^{1} \mathrm{H}$ NMR $\left(250 \mathrm{MHz}, \mathrm{CDCl}_{3}\right): \delta=5.81\left(\mathrm{~s}, 2 \mathrm{H}, \mathrm{CH}_{2}\right), 6.19\left(\right.$ br s, $\left.2 \mathrm{H}, \mathrm{NH}_{2}\right), 6.37(\mathrm{dd}, J=4.0$, $2.6 \mathrm{~Hz}, 1 \mathrm{H}, \mathrm{H}-4), 6.65-6.73$ (m, 2H, H-3', H-5'), 6.93 (br s, 1H, H-5), 7.04 (dd, J=4.0, $1.6 \mathrm{~Hz}$, 1H, H-3), 7.31 (ddd, $J=8.3,7.3,1.2 \mathrm{~Hz}, 1 \mathrm{H}, \mathrm{H}-4^{\prime}$ ), 7.75 (d, $J=8.3 \mathrm{~Hz}, 1 \mathrm{H}, \mathrm{H}-6$ '), 9.54 (d, $J=0.9$ $\mathrm{Hz}, 1 \mathrm{H}, \mathrm{CHO}) ;{ }^{13} \mathrm{C} \mathrm{NMR}\left(63 \mathrm{MHz}, \mathrm{CDCl}_{3}\right): \delta=54.74,110.16,115.64,116.06,117.48,124.61$, 129.83, 131.86, 132.65, 135.04, 150.69, 179.77, 194.03; MS (EI, $70 \mathrm{eV}) \mathrm{m} / \mathrm{z}: 228\left[\mathrm{M}^{+}, 86 \%\right], 133$ (83), 120 (100), 92 (86), 65 (70\%); HRMS-EI Calcd $\mathrm{C}_{13} \mathrm{H}_{12} \mathrm{~N}_{2} \mathrm{O}_{2}$ : 228.0899. Found: 228.0898.

1-[2-(2-Aminophenyl)-2-oxoethyl]-1H-pyrrole-2,4-dicarbaldehyde $\mathbf{( 3 b )}$. Colourless microcrystals (recrystallised from AcOEt-hexane); (0.41 g, 54\%); mp 162-163 ${ }^{\circ} \mathrm{C}$. IR (Nujol, cm $\left.{ }^{-1}\right)$ : 3475, 3375, 1720, 1700, 1680; ${ }^{1} \mathrm{H}$ NMR (400 MHz, $\mathrm{CDCl}_{3}$ ): $\delta=5.84$ (s, 2H, $\mathrm{CH}_{2}$ ), 6.19 (br s, 2H, $\mathrm{NH}_{2}$ ), $6.64-6.70$ (m, 2H, H-3', H-5'), 7.34 (t, J=7.4 Hz, 1H, H-4'), 7.39 (br s, 1H, H-3), 7.53 (br s, $1 \mathrm{H}, \mathrm{H}-5), 7.71$ (d, $J=8.2 \mathrm{~Hz}, 1 \mathrm{H}, \mathrm{H}-6$ '), 9.63 (s, 1H, CHO-2), 9.88 (s, 1H, CHO-4); ${ }^{13} \mathrm{C}$ NMR $\left(100.6 \mathrm{MHz}_{\mathrm{CDCl}}\right): \delta=56.13,109.32,114.28,118.39,119.84,126.45,129.97,133.06,133.77$,

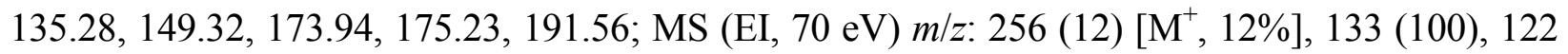
(64), 93 (56) 64 (32\%); HRMS-EI Calcd $\mathrm{C}_{14} \mathrm{H}_{12} \mathrm{~N}_{2} \mathrm{O}_{2}$ : 256.0848. Found: 256.0844.

1-[2-(2-Aminophenyl)-2-oxoethyl]-4-bromo-1H-pyrrole-2-carbaldehyde (3c). Colourless solid after column chromatography [AcOEt-hexane, (1:4)] and recrystallisation (propan-1-ol); (0.50 g, 55\%); mp 179-180 ${ }^{\circ} \mathrm{C}$. IR (Nujol, $\mathrm{cm}^{-1}$ ): 3480, 3380, 1720, 1695; ${ }^{1} \mathrm{H}$ NMR (400 MHz, $\left.\mathrm{CDCl}_{3}\right): \delta$ $=5.76\left(\mathrm{~s}, 2 \mathrm{H}, \mathrm{CH}_{2}\right), 6.19\left(\right.$ br s, $\left.2 \mathrm{H}, \mathrm{NH}_{2}\right), 6.68\left(\mathrm{dd}, J=8.4,1.1 \mathrm{~Hz}, 1 \mathrm{H}, \mathrm{H}-3^{\prime}\right), 6.70$ (ddd, $J=8.1$, 7.1, $\left.1.1 \mathrm{~Hz}, 1 \mathrm{H}, \mathrm{H}-5^{\prime}\right), 6.91$ (dd, $\left.J=1.8,1.1 \mathrm{~Hz}, 1 \mathrm{H}, \mathrm{H}-5\right), 7.01$ (d, $J=1.8 \mathrm{~Hz}, 1 \mathrm{H}, \mathrm{H}-3$ ), 7.32 
(ddd, $\left.J=8.4,7.1,1.2 \mathrm{~Hz}, 1 \mathrm{H}, \mathrm{H}-4^{\prime}\right), 7.69$ (dd, $\left.J=8.1,1.2 \mathrm{~Hz}, 1 \mathrm{H}, \mathrm{H}-6^{\prime}\right), 9.47$ (s, $J=1.1 \mathrm{~Hz}, 1 \mathrm{H}$, $\mathrm{CHO}) ;{ }^{13} \mathrm{C}$ NMR $\left(100.6 \mathrm{MHz}, \mathrm{CDCl}_{3}\right): \delta=54.82\left(\mathrm{CH}_{2}\right), 97.46(\mathrm{C}-4), 115.52\left(\mathrm{C}-1^{\prime}\right), 116.20(\mathrm{C}-$ 5'), 117.62 (C-3'), 125.22 (C-3), 129.76 (C-6'), 131.88 (C-5), 132.08 (C-2), 135.28 (C-4'), 150.82 (C-2'), 179.29 (CHO), 193.14 (CO); MS (EI, 70 eV) m/z: 306 [M+1 , 11\%], 133 (29), 120 (100), 92 (22), 65 (14\%); HRMS-EI Calcd $\mathrm{C}_{13} \mathrm{H}_{11} \mathrm{BrN}_{2} \mathrm{O}_{2}: 306.0004$. Found: 306.0009.

(2E)-3-\{1-[2-(2-aminophenyl)-2-oxoethyl]-2-formyl-1 $H$-pyrrol-3-yl\}- $N, N$-dimethylprop-2-

enamide (3d). Brown-red mirocrystals after column chromatography [EtOAc-hexane, (9:1)] and recrystallisation (AcOEt-hexane); (0.63 g, 65\%); mp 148-150 ${ }^{\circ} \mathrm{C}$; IR (Nujol, cm $\left.{ }^{-1}\right): 3480,3380$, 1680, 1670, 1650; ${ }^{1} \mathrm{H}$ NMR (300 MHz, $\left.\mathrm{CDCl}_{3}\right): \delta=3.00\left(\mathrm{~s}, 3 \mathrm{H}, \mathrm{CH}_{3}\right), 3.10\left(\mathrm{~s}, 3 \mathrm{H}, \mathrm{CH}_{3}\right), 5.72(\mathrm{~s}$, $2 \mathrm{H}, \mathrm{CH}_{2}$ ), 6.12 (br s, 2H, NH${ }_{2}$ ), 6.49 (d, J=2.7 Hz, 1H, H-4), 6.58-6.65 (m, 2H, H-3', H-5'), 6.75 $(\mathrm{d}, J=15.1 \mathrm{~Hz}, 1 \mathrm{H}, \mathrm{H}-\alpha), 6.80(\mathrm{~d}, J=2.7 \mathrm{~Hz}, 1 \mathrm{H}, \mathrm{H}-5), 7.25$ (dd, $\left.J=8.2,1.2 \mathrm{~Hz}, 1 \mathrm{H}, \mathrm{H}-4^{\prime}\right), 7.65$ (dd, $J=8.1,1.2 \mathrm{~Hz}, 1 \mathrm{H}, \mathrm{H}-6$ ) $), 7.90$ (d, $J=15.1 \mathrm{~Hz}, 1 \mathrm{H}, \mathrm{H}-\beta), 9.87$ (s, 1H, CHO); ${ }^{13} \mathrm{C}$ NMR $(75.5$ $\mathrm{MHz}_{\mathrm{CDCl}}$ ): $\delta=35.94,37.40,55.36,107.28,115.52,116.11,117.54,119.13,128.85,129.78$, 131.76, 132.32, 132.93, 135.18, 150.73, 166.49, 178.71, 193.62; MS (EI, $70 \mathrm{eV}) \mathrm{m} / \mathrm{z}: 325\left[\mathrm{M}^{+}\right.$, 67\%], 167 (40), 154 (75), 149 (100), 136 (81), 113 (45), 91 (34), 73 (71), 57 (80\%); HRMS-EI Calcd $\mathrm{C}_{18} \mathrm{H}_{19} \mathrm{~N}_{3} \mathrm{O}_{3}$ : 325.1426. Found: 325.1423 .

1-[2-(2-Aminophenyl)-2-oxoethyl]-1H-pyrrole-2-carboxylic acid methyl ester (3e). Pale green needles after column chromatography [EtOAc-hexane, (1:1] and recrystallisation (propan-2-ol); (0.52 g, 67\%); mp 157-158.5 ${ }^{\circ} \mathrm{C}$; IR (Nujol, $\mathrm{cm}^{-1}$ ): 3485, 3380, 1690, 1670; ${ }^{1} \mathrm{H}$ NMR (400 MHz, $\left.\mathrm{CDCl}_{3}\right): \delta=3.66\left(\mathrm{~s}, 3 \mathrm{H}, \mathrm{CH}_{3}\right), 5.69\left(\mathrm{~s}, 2 \mathrm{H}, \mathrm{CH}_{2}\right), 6.12\left(\mathrm{br} \mathrm{s}, 2 \mathrm{H}, \mathrm{NH}_{2}\right), 6.19(\mathrm{dd}, J=4.0,2.6 \mathrm{~Hz}$, $1 \mathrm{H}, \mathrm{H}-4), 6.58-6.64$ (m, 2H, H-3', H-5'), 6.75 (dd, $J=2.5,1.9 \mathrm{~Hz}, 1 \mathrm{H}, \mathrm{H}-5), 6.98$ (dd, $J=4.0,1.9$ $\mathrm{Hz}, 1 \mathrm{H}, \mathrm{H}-3), 7.22$ (ddd, $\left.J=8.4,7.1,1.3 \mathrm{~Hz}, 1 \mathrm{H}, \mathrm{H}-4^{\prime}\right), 7.68\left(\mathrm{dd}, J=8.1,1.3 \mathrm{~Hz}, 1 \mathrm{H}, \mathrm{H}-6^{\prime}\right) ;{ }^{13} \mathrm{C}$ NMR (100.6 MHz, $\left.\mathrm{CDCl}_{3}\right): \delta=51.52\left(\mathrm{CH}_{3}\right), 55.52\left(\mathrm{CH}_{2}\right), 109.06(\mathrm{C}-4) 116.06(\mathrm{C}-2), 116.46(\mathrm{C}-$ $\left.5^{\prime}\right), 117.94$ (C-3'), 118.56 (C-3), 122.86 (C-1'), 130.17 (C-6'), 130.39 (C-5), 135.38 (C-4'), 151.09 (C-2'), 162.16 (CO ester), 194.96 (CO ketone); MS (EI, $70 \mathrm{eV}) \mathrm{m} / z: 258$ [M+2 25\%], 133 (23), 120 (100), 92 (13\%); HRMS-EI Calcd for $\mathrm{C}_{14} \mathrm{H}_{14} \mathrm{~N}_{2} \mathrm{O}_{3}$ : 258.1004. Found: 258.1008.

1-[2-(2-Aminophenyl)-2-oxoethyl]-4-bromo-1H-pyrrole-2-carboxylic acid methyl ester (3f). Pale yellow microcrystals after recrystallisation (propan-2-ol); $(0.70 \mathrm{~g}, 70 \%) ; \mathrm{mp} 162-164{ }^{\circ} \mathrm{C}$. IR (Nujol, $\mathrm{cm}^{-1}$ ): 3465, 3352, 2924, 1705, 1660; ${ }^{1} \mathrm{H} \mathrm{NMR}\left(300 \mathrm{MHz}, \mathrm{CDCl}_{3}\right): \delta=3.74\left(\mathrm{~s}, 3 \mathrm{H}, \mathrm{CH}_{3}\right)$, 5.73 (s, 2H, CH 2 ), 6.20 (br s, 2H, NH 2 ), 6.66-6.72 (m, 2H, H-3', H-5'), 6.85 (d, J=1.8 Hz, 1H, H3), 7.03 (d, $J=1.8 \mathrm{~Hz}, 1 \mathrm{H}, \mathrm{H}-5), 7.30$ (ddd, $J=8.5,7.9,1.5, \mathrm{~Hz}, 1 \mathrm{H}, \mathrm{H}-4^{\prime}$ ), 7.72 (dd, $J=8.1,1.5$ $\left.\mathrm{Hz}, 1 \mathrm{H}, \mathrm{H}-6{ }^{\prime}\right) ;{ }^{13} \mathrm{C} \mathrm{NMR}\left(75.5 \mathrm{MHz}, \mathrm{CDCl}_{3}\right): \delta=51.34,55.05,96.02,115.39,116.07,117.54$, 119.60, 122.99, 129.18, 129.60, 135.10, 150.70, 160.79, 193,60; MS (ESI) $m / z: 337[\mathrm{M}+\mathrm{H}]^{+}$; HRMS-ESI: $m / z[\mathrm{M}+\mathrm{H}]^{+}$Calcd $\mathrm{C}_{14} \mathrm{H}_{14} \mathrm{BrN}_{2} \mathrm{O}_{3}$ : 337.0188. Found: 337.0192 .

1-[2-(2-Aminophenyl)-2-oxoethyl]-4,5-dibromo-1 $\boldsymbol{H}$-pyrrole-2-carboxylic acid methyl ester (3g). Deep green microcrystals after recrystallisation (propan-2-ol); $(0.80 \mathrm{~g}, 64 \%)$; mp 170-171 ${ }^{\circ} \mathrm{C}$; IR (Nujol, $\mathrm{cm}^{-1}$ ): 3485, 3380, 1690, 1670; ${ }^{1} \mathrm{H}$ NMR (400 MHz, $\left.\mathrm{CDCl}_{3}\right): \delta=3.66\left(\mathrm{~s}, 3 \mathrm{H}, \mathrm{CH}_{3}\right)$, 5.87 (s, 2H, $\mathrm{CH}_{2}$ ), 6.12 (br s, 2H, NH ), 6.58-6.69 (m, 2H, H-3', H-5'), 7.04 (s, 1H, H-3), 7.24 $\left(\mathrm{ddd}, J=8.4,7.8,1.3 \mathrm{~Hz}, 1 \mathrm{H}, \mathrm{H}-4^{\prime}\right), 7.68\left(\mathrm{dd}, J=8.1,1.3, \mathrm{~Hz}, 1 \mathrm{H}, \mathrm{H}-6{ }^{\prime}\right) ;{ }^{13} \mathrm{C} \mathrm{NMR}(100.6 \mathrm{MHz}$, $\left.\mathrm{CDCl}_{3}\right): \delta==51.01\left(\mathrm{CH}_{3}\right), 54.73\left(\mathrm{CH}_{2}\right), 99.94(\mathrm{C}-4) 114.70(\mathrm{C}-5), 115.77\left(\mathrm{C}-1^{\prime}\right), 116.52\left(\mathrm{C}-3^{\prime}\right)$, 
117.99 (C-5'), 120.20 (C-3), 124.55 (C-2), 130.11 (C-6'), 135.62 (C-4'), 151.17 (C-2'), 160.65 (CO ester), 193.15 (CO ketone); MS (EI, 70 eV) m/z: 414 [M+1, 12\%], 337 (24), 120 (100), 92 (15\%); HRMS-EI Calcd for $\mathrm{C}_{14} \mathrm{H}_{12} \mathrm{Br}_{2} \mathrm{~N}_{2} \mathrm{O}_{3}$ : 413.9215. Found: 413.9211.

\section{1-[2-(2-Aminophenyl)-2-oxoehyl]-4-[(1E)-3-(dimethylamino)-3-oxoprop-1-enyl]-1H-pyrrole-}

2-carboxylic acid methyl ester (3h). Colourless microcrystals after column chromatography [EtOAc-hexane, (9:1)] and recrystallisation (AcOEt-hexane); (0.57 g, 54\%); mp 207.5-209 ${ }^{\circ} \mathrm{C}$. IR (Nujol, $\mathrm{cm}^{-1}$ ): 3410, 3310, 1730, 1690, 1650; ${ }^{1} \mathrm{H}$ NMR (400 MHz, DMSO-d $\left.d_{6}\right): \delta=2.96$ (s, 3H, $\left.\mathrm{CH}_{3}\right), 3.18\left(\mathrm{~s}, 3 \mathrm{H}, \mathrm{CH}_{3}\right), 3.72\left(\mathrm{~s}, 3 \mathrm{H}, \mathrm{CH}_{3}\right.$ ester), $5.86\left(\mathrm{~s}, 2 \mathrm{H}, \mathrm{CH}_{2}\right), 6.66$ (ddd, J=8.4, 8.2, $1.0 \mathrm{~Hz}$, $1 \mathrm{H}, \mathrm{H}-5^{\prime}$ ), 6.85 (dd, $\left.J=8.4,1.0 \mathrm{~Hz}, 1 \mathrm{H}, \mathrm{H}-3^{\prime}\right), 6.98$ (d, $\left.J=15.2 \mathrm{~Hz}, 1 \mathrm{H}, \mathrm{H}-\alpha\right), 7.20$ (br s, 2H, $\mathrm{NH}_{2}$ ), 7.36 (ddd, $J=8.4,8.2,1.3 \mathrm{~Hz}, 1 \mathrm{H}, \mathrm{H}-4$ '), 7.40 (d, $\left.J=15.2 \mathrm{~Hz}, 1 \mathrm{H}, \mathrm{H}-\beta\right), 7.43$ (d, $J=1.8$ $\mathrm{Hz}, 1 \mathrm{H}, \mathrm{H}-5), 7.52$ (d, $J=1.8 \mathrm{~Hz}, 1 \mathrm{H}, \mathrm{H}-3), 7.90$ (dd, $\left.J=8.2,1.3 \mathrm{~Hz}, 1 \mathrm{H}, \mathrm{H}-6{ }^{\prime}\right) ;{ }^{13} \mathrm{C}$ NMR $(100.6$ MHz, DMSO-d $\left.d_{6}\right): \delta=35.61\left(\mathrm{CH}_{3}\right), 37.10\left(\mathrm{CH}_{3}\right), 51.43\left(\mathrm{CH}_{3}\right), 55.72\left(\mathrm{CH}_{2}\right), 114.65(\mathrm{C}-4), 114.88$ (C-5'), 115.15 (C- $\alpha$ ), 115.72 (C-5), 117.42 (C-3'), 120.23 (C-2), 123.35 (C-1'), 130.61 (C-6'), 132.68 (C-3), 134.56 (C- $\beta$ ), 135.03 (C-4'), 151.62 (C-2'), 161.00 (CO, ester), 166.39 (CO, amide), 194.54 (CO); MS (EI, $70 \mathrm{eV})$ m/z: 355 [M+, 13\%], 310 (29), 223 (32), 120 (100), 92 (17\%); HRMS-EI Calcd $\mathrm{C}_{19} \mathrm{H}_{21} \mathrm{~N}_{3} \mathrm{O}_{4}: 355.1532$. Found: 355.1536.

Formylation of $(E)-3-[1-(4-m e t h y l p h e n y l s u l f o n y l)-1 H$-pyrrol-3-yl]- $N, N$-dimethylprop-2enamide. A stirred mixture of prop-2-enamide $4^{12}(0.50 \mathrm{~g}, 1.57 \mathrm{mmol})$ and $\mathrm{AlCl}_{3}(0.49 \mathrm{~g}, 3.71$ $\mathrm{mmol})$ in dry 1,2-dichloroethane $(10 \mathrm{~mL})$ was cooled to $-40^{\circ} \mathrm{C}$ under argon. Dichloromethylether ( $0.25 \mathrm{~g}, 2.20 \mathrm{mmol})$ was added dropwise and then the temperature was allowed to reach $-10^{\circ} \mathrm{C}$. Stirring was continued for $1 \mathrm{~h}$ and then the reaction mixture was added to ice-water, the organic layer separated and the aqueous layer extracted with $\mathrm{CH}_{2} \mathrm{Cl}_{2}(3 \times 10 \mathrm{~mL})$. The combined organic extracts were washed with a $10 \% \mathrm{w} / \mathrm{v}$ aqueous solution of $\mathrm{NaHCO}_{3}(3 \times 10 \mathrm{~mL})$, brine $(20 \mathrm{~mL})$ and dried $\left(\mathrm{MgSO}_{4}\right)$. The solvent was removed under reduced pressure and the residue was purified by column chromatography $\left(\mathrm{CH}_{2} \mathrm{Cl}_{2}\right.$-acetone, $90: 1$ to $\left.1: 1\right)$ to give in the first fraction compound 6 $(0.06 \mathrm{~g}, 11 \%)$ and in the second fraction compound $5(0.29 \mathrm{~g}, 53 \%)$.

\section{(E)-3-\{2-formyl-[1-(4-methylphenylsulfonyl]-1 $\boldsymbol{H}$-pyrrol-3-yl\}- $\mathbf{N}, \boldsymbol{N}$-dimethylprop-2-enamide}

(5). Pale yellow microcrystals after recrystallisation (AcOEt-hexane); (0.29 g, 53\%); mp 155-157 ${ }^{\circ} \mathrm{C}$ (dec); IR (Nujol, $\left.\mathrm{cm}^{-1}\right): 1680,1660,1330,1120 ;{ }^{1} \mathrm{H}$ NMR $\left(300 \mathrm{MHz}, \mathrm{CDCl}_{3}\right): \delta=2.36(\mathrm{~s}, 3 \mathrm{H}$, $\mathrm{CH}_{3}$ ), 3.07 (br s, $6 \mathrm{H}, 2 \times \mathrm{CH}_{3}$ ), 6.58 (br s, 1H, H-4), 6.84 (d, $J=15.2 \mathrm{~Hz}, 1 \mathrm{H}, \mathrm{H}-\alpha$ ), 7.31 (d, $J=8.1$ Hz, 2H, H-3', H-5') 7.57 (br s, 1H, H-5), 7.76 (d, J=8.1 Hz, H-2', 2H, H-6'), 7.94 (d, J= 15.2 $\mathrm{Hz}, 1 \mathrm{H}, \mathrm{H}-\beta), 10.20(\mathrm{~s}, 1 \mathrm{H}, \mathrm{CHO}) ;{ }^{13} \mathrm{C} \mathrm{NMR}\left(75.5 \mathrm{MHz}, \mathrm{CDCl}_{3}\right): \delta=21.7,35.8,37.5,110.2$, 123.6, 127.5, 128.8, 129.2, 130.3, 131.8, 134.8, 135.0, 146.2, 166.1, 179.4; MS (EI, $70 \mathrm{eV}) \mathrm{m} / \mathrm{z}$ : $346\left[\mathrm{M}^{+}, 10 \%\right], 307$ (25), 154 (100), 136 (89), 107 (54), 91 (38), 81 (36), 69 (54), 57 (73\%); HRMS-EI Calcd for $\mathrm{C}_{17} \mathrm{H}_{18} \mathrm{~N}_{2} \mathrm{O}_{4} \mathrm{~S}:$ 346.0987. Found: 346.0985 .

\section{(E)-3-\{5-formyl-[1-(4-methylphenylsulfonyl]-1 H-pyrrol-3-yl\}-N, $\boldsymbol{N}$-dimethylprop-2-enamide}

(6). Pale yellow microcrystals after recrystallisation (AcOEt-hexane); (0.06 g, 11\%); mp 153-154 ${ }^{\circ} \mathrm{C}$ (dec); IR (Nujol, $\left.\mathrm{cm}^{-1}\right): 1690,1670,1340,1150 ;{ }^{1} \mathrm{H}$ NMR $\left(300 \mathrm{MHz}, \mathrm{CDCl}_{3}\right): \delta=2.41(\mathrm{~s}, 3 \mathrm{H}$, $\mathrm{CH}_{3}$ ), 3.05 (d, 6H, $2 \times \mathrm{CH}_{3}$ ), 6.70 (d, $J=15.4 \mathrm{~Hz}, 1 \mathrm{H}, \mathrm{H}-\alpha$ ), 7.29 (br s, 1H, H-4), 7.29-7.36 (m, 
2H, H-3', H-5'), 7.46 (d, J=15.4 Hz, 1H, H- $\beta$ ), 7.69 (br s, 1H, H-2), 7.75-7.81 (m, 2H, H-2', H6'), 10.00 (s, 1H, CHO); ${ }^{13} \mathrm{C}$ NMR (75.5 MHz, $\left.\mathrm{CDCl}_{3}\right): \delta=21.8,36.1,37.8,118.8,122.8,127.5$, 128.6, 130.2, 132.3, 134.5, 134.9, 146.3, 166.1, 179.2; MS (EI, $70 \mathrm{eV}) \mathrm{m} / z: 346$ [M ${ }^{+}, 52 \%$ ], 307 (35), 289 (20), 154 (100), 136 (95), 120 (24), 107 (41), 89 (38), 77 (39), 65 (20\%); HRMS-EI Calcd $\mathrm{C}_{17} \mathrm{H}_{18} \mathrm{~N}_{2} \mathrm{O}_{4} \mathrm{~S}: 346.0987$. Found: 346.0983 .

General procedure for the detosylation of $(E)-3-\{2$ or 5-formyl-[1-(4-methylphenylsulfonyl]$1 \mathrm{H}$-pyrrol-4 or 3-yl\}-N,N-dimethylprop-2-enamides $(5,6)$

A mixture of prop-2-enamides $5(0.22 \mathrm{~g}, 0.64 \mathrm{mmol})$ or $6(0.06 \mathrm{~g}, 0.17 \mathrm{mmol})$ and $\mathrm{K}_{2} \mathrm{CO}_{3}(0.20 \mathrm{~g}$, $1.45 \mathrm{mmol}) \sigma \varepsilon$ dry $\mathrm{MeOH}(15 \mathrm{~mL})$ under argon was stirred for $1 \mathrm{~h}$. The reaction mixture was poured into brine $(30 \mathrm{~mL})$, extracted with EtOAc $(3 \times 15 \mathrm{~mL})$, and dried $\left(\mathrm{Na}_{2} \mathrm{SO}_{4}\right)$. The solvent was removed under vacuo and the crude product $\mathbf{2 d}$ or 7 was purified by recrystallisation.

(E)-3-[2-formyl-1H-pyrrol-3-yl]- $\mathbf{N}, \mathbf{N}$-dimethylprop-2-enamide (2d). Brown microcrystals after recrystallisation (EtOAc-hexane); (0.11 g, 88\%); mp 123-124 ${ }^{\circ} \mathrm{C}(\mathrm{dec})$; IR (Nujol, $\left.\mathrm{cm}^{-1}\right)$ : 1690, 1670; ${ }^{1} \mathrm{H}$ NMR $\left(300 \mathrm{MHz}, \mathrm{CDCl}_{3}\right): \delta=3.03\left(\mathrm{~s}, 3 \mathrm{H}, \mathrm{CH}_{3}\right), 3.09\left(\mathrm{~s}, 3 \mathrm{H}, \mathrm{CH}_{3}\right), 6.45-6.51(\mathrm{~m}, 1 \mathrm{H}, \mathrm{H}-$ 4), 6.77 (d, $J=15.2 \mathrm{~Hz}, 1 \mathrm{H}, \mathrm{H}-\alpha), 7.03-7.12$ (m, 1H, H-5), 7.90 (d, J=15.2 Hz, 1H, H- $\beta$ ), 9.80 (s, $1 \mathrm{H}, \mathrm{CHO}), 9.81$ (br s, $1 \mathrm{H}, \mathrm{NH}) ;{ }^{13} \mathrm{C} \mathrm{NMR}\left(75.5 \mathrm{MHz}, \mathrm{CDCl}_{3}\right): \delta=34.9,36.7,112.5,124.7,126.7$, 129.2, 131.8, 135.0, 165.4, 181.6; MS (EI, $70 \mathrm{eV}) \mathrm{m} / z$ : 192 [M+25\%], 148 (65), 120 (100), 92 (40), 65 (41\%); HRMS-EI Calcd $\mathrm{C}_{10} \mathrm{H}_{12} \mathrm{~N}_{2} \mathrm{O}_{2}$ : 192.0899. Found: 192.0898.

(E)-3-[5-Formyl-1 $H$-pyrrol-3-yl]- $\mathbf{N}, \mathbf{N}$-dimethylprop-2-enamide (7). Brown microcrystals after recrystallisation (AcOEt-hexane); $(0.027 \mathrm{~g}, 82 \%) ; \mathrm{mp} \mathrm{129-130}{ }^{\circ} \mathrm{C}(\mathrm{dec})$; IR (Nujol, $\left.\mathrm{cm}^{-1}\right): 1680$, 1670; ${ }^{1} \mathrm{H}$ NMR $\left(300 \mathrm{MHz}, \mathrm{CDCl}_{3}\right): \delta=3.11\left(\mathrm{~s}, 3 \mathrm{H}, \mathrm{CH}_{3}\right), 3.12\left(\mathrm{~s}, 3 \mathrm{H}, \mathrm{CH}_{3}\right), 6.66(\mathrm{~d}, J=15.4 \mathrm{~Hz}$, $1 \mathrm{H}, \mathrm{H}-\alpha), 7.14$ (s, 1H, H-4), 7.31 (s, 1H, H-2), 7.61 (d, 1H, J=15.4 Hz, H- $\beta$ ), 9.54 (s, 1H, CHO), 9.99 (br s, $1 \mathrm{H}, \mathrm{NH}) ;{ }^{13} \mathrm{C}$ NMR $\left(75.5 \mathrm{MHz}, \mathrm{CDCl}_{3}\right): \delta=34.8,36.7,117.8,122.6,129.9,133.3$, 132.35, 136.3, 165.8, 181.8; MS (ESI) $m / z$ : $193.1[\mathrm{M}+\mathrm{H}]^{+}$; HRMS-ESI $[\mathrm{M}+\mathrm{H}]^{+}$Calcd $\mathrm{C}_{10} \mathrm{H}_{13} \mathrm{~N}_{2} \mathrm{O}_{2}$ : 193.0977. Found: 193.0981 .

( \pm )-1-(2-Aminophenyl)-2-[4-bromo-2-(hydroxymethyl)-1H-pyrrol-1-yl]ethanol (9). To a stirred solution of 1-[2-(2-aminophenyl)-2-oxoethyl]-4-bromo-2-formyl-1H-pyrrole 3c $(0.20 \mathrm{~g}$, $0.66 \mathrm{mmol})$ in dry ether $(10 \mathrm{~mL})$, sodium borohydride $(0.048 \mathrm{~g}, 1.27 \mathrm{mmol})$ was added and the reaction mixture stirred at room temperature for $48 \mathrm{~h}$. The solvent was evaporated, water $(15 \mathrm{~mL})$ was added and acidified with glacial acetic acid to $\mathrm{pH}=5$. The mixture was extracted with dichloromethane $(3 \times 5 \mathrm{~mL})$, the combined organic phases dried $\left(\mathrm{Na}_{2} \mathrm{SO}_{4}\right)$ and evaporated to give an oil (0.14 g, 68\%); IR (Nujol, $\mathrm{cm}^{-1}$ ): 3640, 3440, 3340; ${ }^{1} \mathrm{H}$ NMR (250 MHz, DMSO- $\left.d_{6}\right): \delta 3.99$ $(\mathrm{s}, 1 \mathrm{H}, \mathrm{CH}), 4.00(\mathrm{~s}, 1 \mathrm{H}, \mathrm{CH}), 4.37\left(\mathrm{~d}, 2 \mathrm{H}, \mathrm{CH}_{2}\right), 5.11\left(2 \mathrm{H}, \mathrm{CH}_{2}\right) 5.20(1 \mathrm{H}, \mathrm{CH}), 5.57$ (br s, 1H, H5), 5.99 (br s, 1H, H-3), 6.55 (t, 1H, H-5'), 6.65 (d, 1H, H-3'), 6.97 t, 1H, H-4'), 7.14 (d, J = 7.5 $\left.\mathrm{Hz}, 1 \mathrm{H}, \mathrm{H}-6{ }^{\prime}\right) ;{ }^{13} \mathrm{C}$ NMR (63 MHz, DMSO- $\left.d_{6}\right): \delta$ 49.78, 52.88, 68.24, 90.70, 107.86, 113.63, 114.48, 120.60, 123.96, 125.08, 126.26, 132.35, 143.74; MS (EI, 70 eV) m/z: 310 [M+1 19\%], 292 (18), 189 (32), 171 (46), 122 (100\%); HRMS-EI Calcd $\mathrm{C}_{13} \mathrm{H}_{15} \mathrm{BrN}_{2} \mathrm{O}_{2}$ : 310.0317. Found 310.0312 . 


\section{Acknowledgements}

This research was co-funded by the European Union in the framework of the program "Pythagoras I" of the "Operational Program for Education and Initial Vocational Training" of the $3^{\text {rd }}$ Community Support Framework of the Hellenic Ministry of Education, funded by $25 \%$ from national sources and by $75 \%$ from the European Social Fund (ESF).

We are particularly grateful to A. Cakebread and R. Tye, King's College London, for mass spectra obtained on machines funded by the University of London, Dr. F. Sakellaridis and Dr. A. Badeka for mass spectra and Dr. V. Exarchou for NMR spectra on machines funded by the Horizontal Laboratory and Unit Centres of the University of Ioannina.

\section{References and Notes}

1. Marfil, M.; Albericio, F.; Álvarez, M. Tetrahedron 2004, 60, 8659.

2. (a) Hinze, C.; Kreipl, A. Terpin, A.; Steglich, W. Synthesis 2007, 608. (b) Liu, J.-H.; Yang, Q.-C.; Mak, T. C. W.; Wong, H. N. C. J. Org. Chem. 2000, 65, 3587.

3. Belanger, P. C.; Atkinson, J. G.; Rooney, C. S.; Britcher, S. F.; Remy, D. C. J. Org. Chem. 1983, 48, 3234.

4. Campiani, G.; Butini, S.; Gemma, S.; Nacci, V.; Fattorusso, K.; Catalanotti, B.; Ciorgi, G.; Cagnotto, A.; Goegan, M.; Mennini, T.; Minetti, P.; Di Cesare, M. A.; Mastroianni, D.; Scaffeta, N.; Galletti, B.; Stasi, M. A.; Castorina, M.; Pacifici, L.; Ghirardi, O.; Tinti, O.; Carminati, P. J. Med. Chem. 2002, 45, 344.

5. Artico, M.; Corelli, F.; Massa, S.; Stefanich, G. Synthesis 1983, 931.

6. Campiani, G.; Nacci, V.; Bechelli, S.; Ciani, S. M.; Garofalo, A.; Fiorini, I.; Wikstrom, H.; De Boer, P.; Liao, Yi.; Tepper, P. G.; Cagnotto, A., Mennini, T. J. Med. Chem. 1998, 41, 3763.

7. Andreani, A.; Leoni, A.; Rambaldi, M.; Locatelli, A.; Bossa, R. Eur. J. Med. Chem. 1997, $32,151$.

8. Grehm, L. Chem. Scr. 1979, 13, 78.

9. Silverstein, R. M.; Ryskiewicz, E. E.; Willard, C. Org. Synth. Coll. 4, 831.

10. Anderson, H. A.; Loader, C. E.; Foster A. Can. J. Chem. 1980, $58,2527$.

11. Sonnet, P. E. J. Org. Chem. 1971, 36, 1005.

12. Karousis, K.; Liebscher, J.; Varvounis, G. Synthesis 2006, 1494.

13. (a) Harbuck, J. W.; Rapoport, H. J. Org. Chem. 1972, 37, 3618. (b) Banwell, M. G.; Hamel, E.; Hockless, D. C. R.; Verdier-Pinard, P.; Willis, A. C.; Wong, D. J. Bioorg. Med. Chem. 2006, 14, 4627.

14. Anderson, H. J.; Lee, S.-F. Can. J. Chem. 1965, 43, 409.

15. Behrens, C.; Christoffersen, M. W.; Gram, L.; Nielsen, P. H. Bioorg. Med. Chem. Lett. 1997, 7, 321.

16. Barker, P.; Gendler, P.; Rapoport, H. J. Org. Chem., 1978, 43, 4849. 